
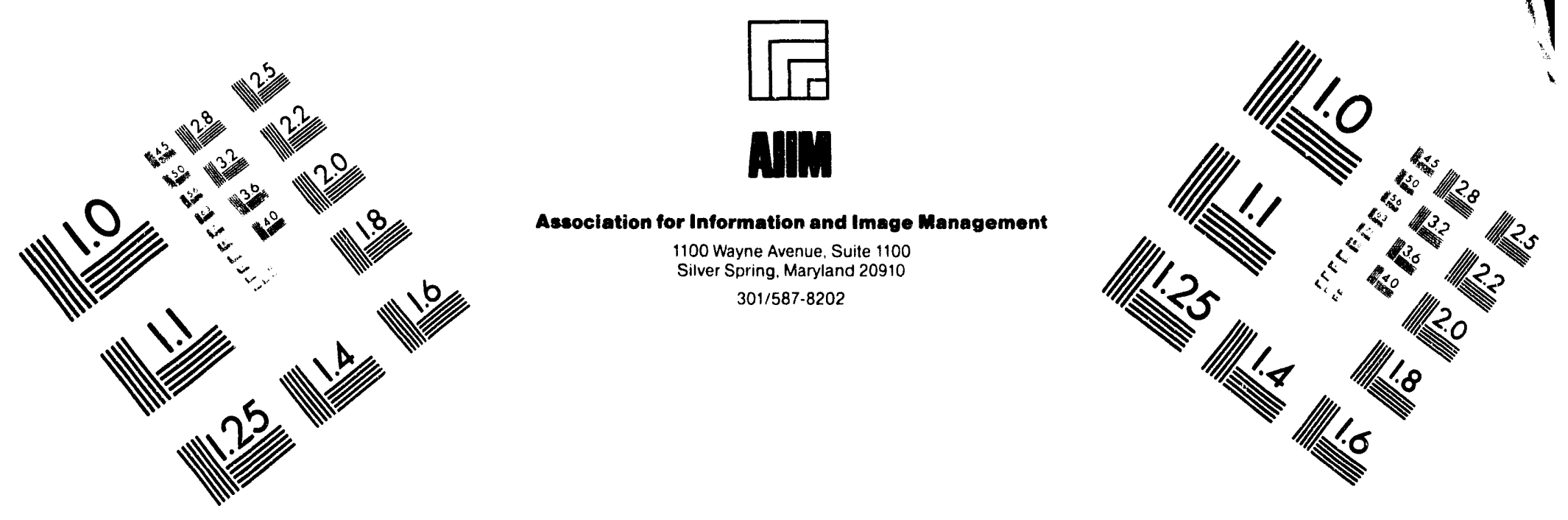

\title{
Centimeter
}

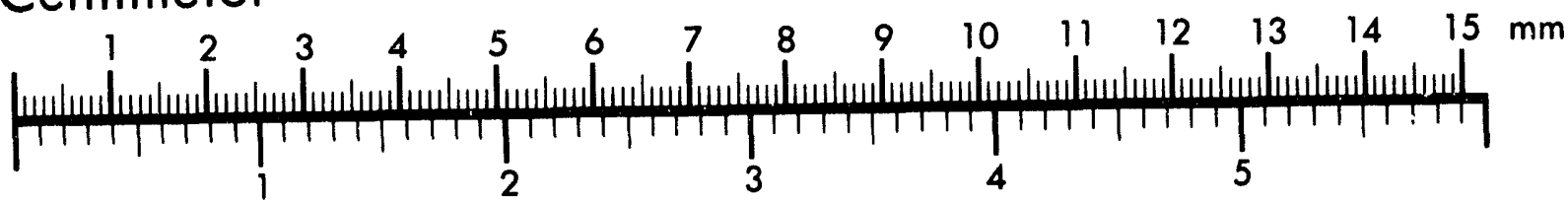
Inches
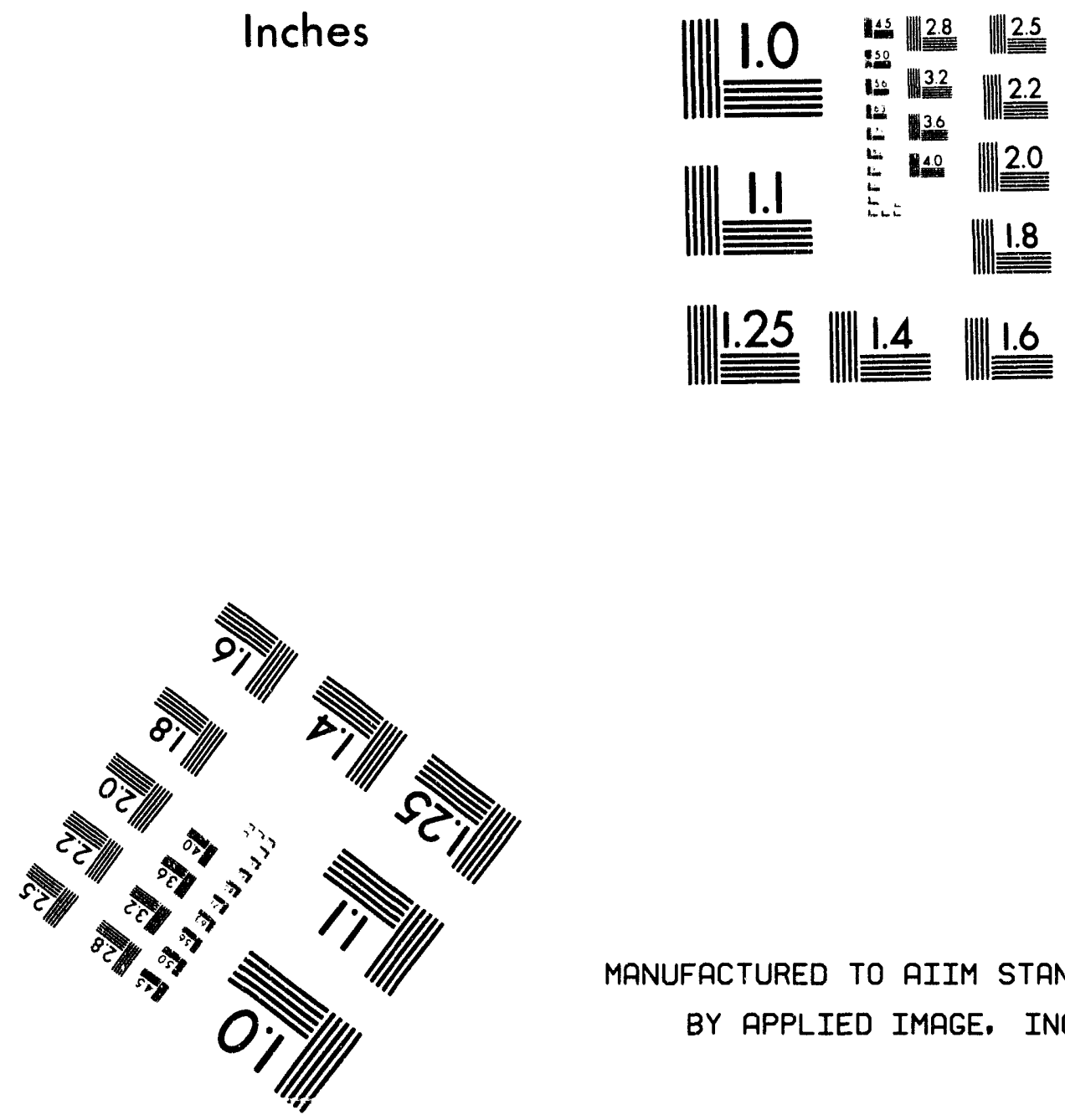

MANUFACTURED TO AIIM STANDARDS

BY APPLIED IMAGE, INC.

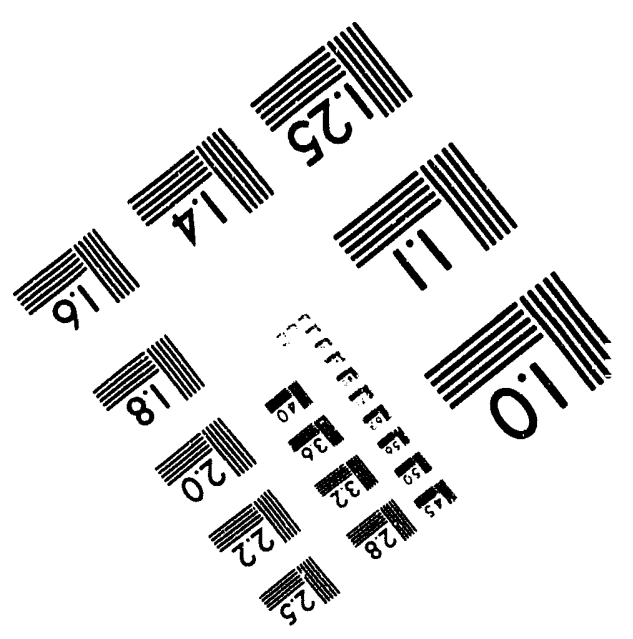



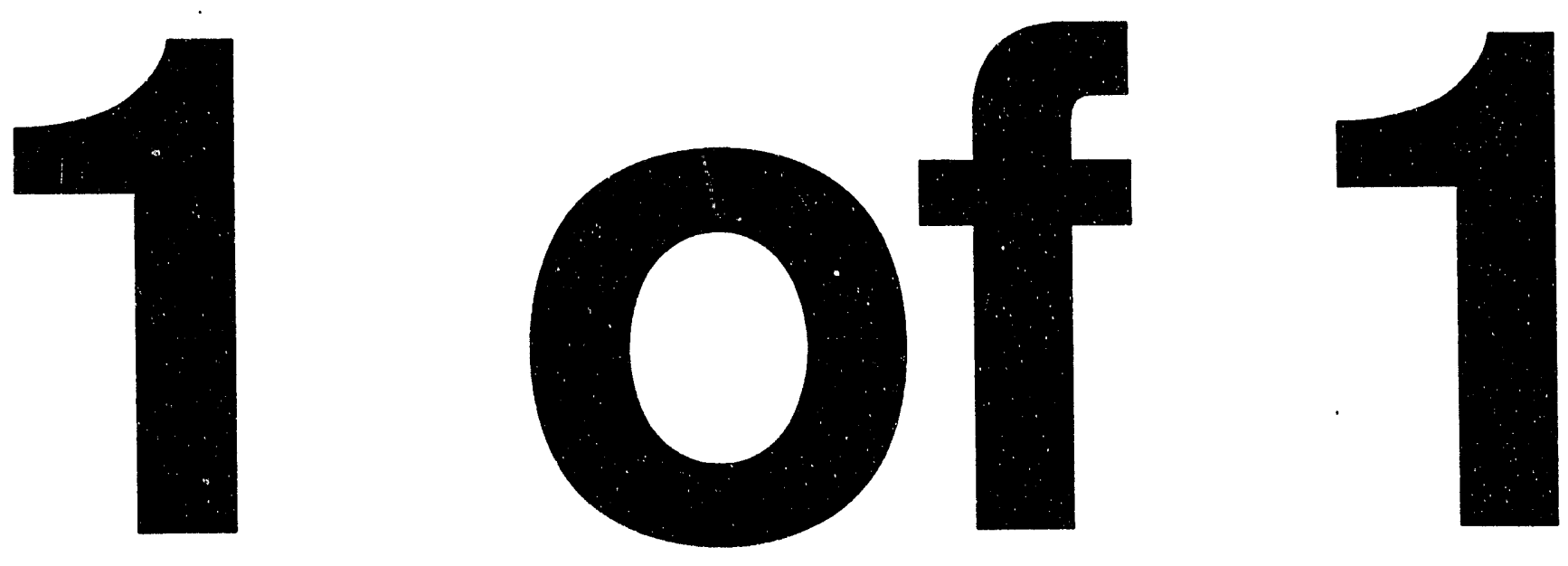


\title{
Membrane Function in Lipid Mutants of Arabidopsis
}

\author{
First Year Progress Report on Department of Energy Grant DE-FG06-92ER20077. June 1993
}

Summary: The major achievements from the first year of this grant include the completion of work on the biochemical characterization of the fad3 mutants deficient in 18:3 fatty acid synthesis and the fab2 mutant that accumulates increased amounts of 18:0. Studies of the cell biology and physiology of the fab2 and fad2 mutants have provided evidence for some of the critical roles played by unsaturated fatty acids as components of plant membranes. Finally, the fab2 mutant has allowed us to carry out the first isolation and characterization of intergenic suppressor mutations in a higher plant.

\section{Biochemical analysis}

The biogenesis of the highly unsaturated membranes that are typical of higher plants is largely dependent on the sequential conversion of 18:1 esterified in membrane glycerolipids to $18: 2$ and 18:3. Our previous genetic and biochemical studies characterized two genes in Arabidopsis, fadC and fadD, that control desaturation of $18: 1$ to $18: 2$ and $18: 2$ to $18: 3$ respectively as well as desaturation of the equivalent 16-carbon fatty acids. The fadC and fadD loci are believed to be structural genes encoding chloroplast proteins that correspond to the enzyme activities observed in chloroplast envelope preparations. On the other hand, the fad2 locus controls a microsomal desaturase that is responsible for conversion of 18:1 to 18:2 on phospholipids in leaves, roots and seeds of Arabidopsis plants. In the present work, we have studies an Arabidopsis mutant that appears to be deficient in the major extrachloroplast 18:2 desaturase that is the companion enzyme to the 18:1 desaturase controlled by the fad 2 gene.

The overall fatty compositions of leaf and root lipids from the fad 3 mutants were characterized by reduced levels of 18:3 and correspondingly elevated levels of $18: 2$ as a consequence of a single nuclear mutation. Comparison of the fatty acid compositions of individual lipids from wild type and mutant plants showed that chloroplast lipids were largely unaffected by the mutation, whereas each of the phospholipids synthesized on the endoplasmic reticulum in the mutant exhibited a marked reduction in the proportion of 18:3 relative to wild type. These and other results indicate that the fad 3 mutants are deficient in the activity of an endoplasmic reticulum 18:2 desaturase. In root tissues, this enzyme appears to account for over $80 \%$ of the 18:3 present. However, in young leaves of the mutant, phosphatidylcholine (the major phospholipid) contains 16\% 18:3 compared with 29\% in the wild type, and the proportion of 18:3 in this lipid increases with plant age in both wild type and mutant plants. These results reflect an exchange of lipid between the chloroplast and endoplasmic reticulum that allows the chloroplast desaturases to provide highly unsaturated lipids to the extrachloroplast membranes of leaf cells. A paper describing this work has been accepted for publication in the Journal of Biological Chemistry and a copy is attached. A similar approach has been taken in studying the biochemistry of lipid synthesis in the fab2 mutant and this work is now being prepared for publication.

\section{Physiology of the fad2 Mutant}

The fad2 mutants of Arabidopsis have been characterized as being deficient in activity of the microsomal 18:1 desaturase that is responsible for production of pclyunsaturated lipids on the eukaryotic pathway of lipid synthesis. Available evidence suggests that the FAD2 locus is the structural gene for the desaturase. A second 16:1/18:1 desaturase (controlled by the FADC locus) is located in the chloroplasts of Arabidopsis leaves and is able to desaturate all the major chloroplast glycerolipids. For this reason, the biochemical effect of the fad2 lesion is largely

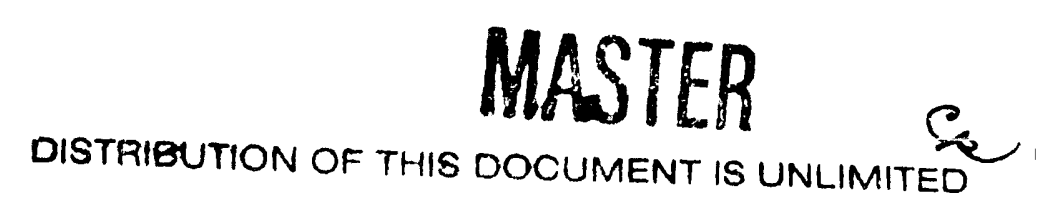


confined to the extrachloroplast membranes of the cell. Furthermore, exchange of lipids between the chloroplast and endoplasmic reticulum leads to the appearance of significant amounts of $18: 3$ (but not 18:2) in the extrachloroplast membranes of leaves of fad 2 mutant plants. Phosphatidylcholine, the most abundant extra chloroplast lipid contained $2 \% 18: 2$ and $14 \% 18: 3$ in leaves of fad2.2 plants used in this study compared with wild type levels of $33 \%$ and $40 \%$ respectively. In tissues (such as roots) that lack chloroplasts, the effects of the mutation are greater. Roots of fad2 plants contained $7-9 \% 18: 2+18: 3$ compared with $65 \%$ in wild type.

The fad2 mutants showed growth characteristics at $22^{\circ} \mathrm{C}$ that were very similar to wild type. By contrast, at $12^{\circ} \mathrm{C}$, the mutants failed to undergo stem elongation during reproductive growth although they produced normal flowers and fertile seeds. After transfer to $6^{\circ} \mathrm{C}$, rosette leaves of the mutants gradually died and the plants were inviable.

While the deficiency in polyunsaturated lipids has severe consequence in the long term, three lines of evidence suggest that the change(s) in membrane function in fad 2 plants at $6^{\circ} \mathrm{C}$ only begin to compromise cell viability after (at least) several days. The appearance of visual symptoms occurs gradually in fad2-2 plants over a period of 10 to 14 days at low temperature. Furthermore, fad2-2 plants that were returned to $22^{\circ} \mathrm{C}$ after three days exposure at $6^{\circ} \mathrm{C}$ recovered fully and were not appreciably slower in growth or development than wild type plants subjected to the same treatment. Finally, one allele at the fad2 locus (fad2-1) produces a leaky mutation at $6^{\circ} \mathrm{C}$. After transfer from $22^{\circ} \mathrm{C}$ to $6^{\circ} \mathrm{C}$ the glycerolipids of fad2. 1 plants reverted to a nearly wild type fatty acid composition over a period of two weeks. Despite the gradual nature of this change in lipid polyunsaturation, fad2-1 plants remained healthy at $6^{\circ} \mathrm{C}$ and grew as rapidly as wild type plants at this temperature.

The hypothesis that an acute loss of membrane integrity at low temperatures, as a result of a phase change within the lipid bilayer, constitutes the primary event leading to injury and death of chilling sensitive plants has been a dominant theme in considering the functional relevance of glycerolipid unsaturation in higher plants. We consider it unlikely that any such major disruption of membrane structure is involved in producing the phenotypes described here for fad2 mutants of Arabidopsis. Thus, the liquid-crystalline phase to gel phase transition temperatures for 18:1/18:1- and 16:0/18:1-phosphatidylcholines (the major lipid species in extrachloroplast membranes of fad2 plants) are $-17^{\circ} \mathrm{C}$ and $-3^{\circ} \mathrm{C}$, respectively. In contrast, the reduction in stem elongation was observed in fad2 plants at $12^{\circ} \mathrm{C}$, apparently in the absence of any major disruption of plant metabolism. Furthermore, the onset of damage at $6^{\circ} \mathrm{C}$ was gradual and chronic. These observations are clearly not consistent with a gross disruption of membrane structure, but instead suggest limited defects in membrane function. Available evidence indicates that the action of the FAD2 gene product is restricted to desaturation of $18: 1$ esterified in membrane glycerolipids $(10,15,16)$ so we have considered our findings in the context of membrane lipid function. Nevertheless, we cannot rule out the possibility that the lethal consequences of the fad2 miltations reflect a requirement for polyunsaturated fatty acids (derived from membrane lipids) in other cellular processes.

\section{The Dwarf fab2 Mutant}

The Arabidopsis fab2 mutant was originally isolated in a screen of seed fatty acid composition in which it was identified as having a three-fold increase in seed stearate (18:0) relative to wild type. However, the most striking feature of the fab2 mutant is not its lipid composition but an extreme dwarf growth habit with mutant plants growing to less than one-tenth of the size of the wild type. Although the dwarf phenotype is extreme the mutant retains normal body pattern and chronology of development. The number of rosette leaves at the onset of flowering was the same in wild type and mutant plants. The dwarf size of fab2 plants is a consequence of reduced expansion of many, but not all, cell types. The area of leaf epidermal cells is reduced almost 
10-fold in the fab2 mutant. The average cross-sectional area of the palisade parenchyma cells is reduced five-fold in the mutant, and this reduction in size is associated with a failure to develop the characteristic leaf anatomy evident in the wild type. In contrast, stomatal guard cells and trichomes of the fab2 leaves are morphologically normal.

As a first test of the possibility that the dwarf phenotype was unrelated to the altered lipid composition, we analyzed 223 segregating $F_{2}$ progeny from a cross of fab2 $x$ wild type and found perfect cosegregation of the two characters. To further investigate the relationship between the high 18:0 levels of fab2 plants and the dwarf phenotype, we screened a remutagenized population derived from the fab2 line for plants in which the dwarf phenotype was suppressed. From a screen of $20,000 \mathrm{M}_{1}$ plants, one individual, R61, was identified as having a putative wild type growing sector. The $M_{2}$ progeny derived from self-pollination of this sector were scored for growth habit and leaf fatty acid composition. Three classes of progeny were found (wild type growth, < 3\% 18:0; intermediate size, 4-8\% 18:0; dwarf growth > 10\% 18:0) and the frequency distribution for these classes was a good fit to the $1: 2: 1$ hypothesis. Also, of 102 $\mathrm{F}_{2}$ progeny from a cross between a normal growing $\mathrm{M}_{2}$ plant (homozygous suppressor) and wild type, 6 displayed the dwarf phenotype - a proportion consistent with the Mendelian segregation of two unlinked loci. These data indicate that the second mutation in R61 is a semidominant intergenic suppressor that is unlinked to the fab2 locus. The observation that the suppressor mutation simultaneously restores normal lipid composition and normal growth provides definitive evidence that the two traits are causally related. At least three other suppressor mutants have been identified by screening the $M_{2}$ population.

The mechanisms through which physical parameters determine the function of biological membranes are poorly understood. However, with respect to the fatty acid components of membrane glycerolipids, it is generally accepted that three interrelated features are of primary importance: the phase behavior of the lipid, the fluidity characteristic contributed to the lipid bilayer, and the shape of the molecule. These characteristics are influenced by the identity of the lipid head group but, in all cases, the thermodynamic behavior is such that the physical effects of increased stearate in membrane lipids would be predicted to be offset by increased temperature. Growth temperatures in the range $15^{\circ}-33^{\circ} \mathrm{C}$ did not alter the appearance of fab2 plants but at $36^{\circ} \mathrm{C}$ the dwarf phenotype was substantially corrected. Indeed, the mutant appeared to grow better than wild type Arabidopsis at this temperature. The internal structure of mutant leaves was also restored to normal at $36^{\circ} \mathrm{C}$. In contrast, the 18:0 content of leaves of fab2 plants grown at $36^{\circ} \mathrm{C}$ was the same as that of mutant plants grown at $22^{\circ} \mathrm{C}$. Our results therefore suggest the possibility that the consequences of high 18:0 on fab2 plants may be mediated through an effect on membrane structure.

The most interesting aspect of this study is that it provides an example in which altered levels of a cell structural component produce a dwarf phenotype that is at least as profound as those caused by mutations in known developmental processes.

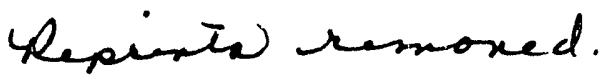

\title{
DISCLAIMER
}

\begin{abstract}
This report was prepared as an account of work sponsored by an agency of the United States Government. Neither the United States Government nor any agency thereof, nor any of their employees, makes any warranty, express or implied, or assumes any legal liability or responsibility for the accuracy, completeness, or usefulness of any information, apparatus, product, or process disclosed, or represents that its use would not infringe privately owned rights. Reference herein to any specific commercial product, process, or service by trade name, trademark, manufacturer, or otherwise does not necessarily constitute or imply its endorsement, recommendation, or favoring by the United States Government or any agency thereof. The views and opinions of authors expressed herein do not necessarily state or reflect those of the United States Government or any agency thereof.
\end{abstract}



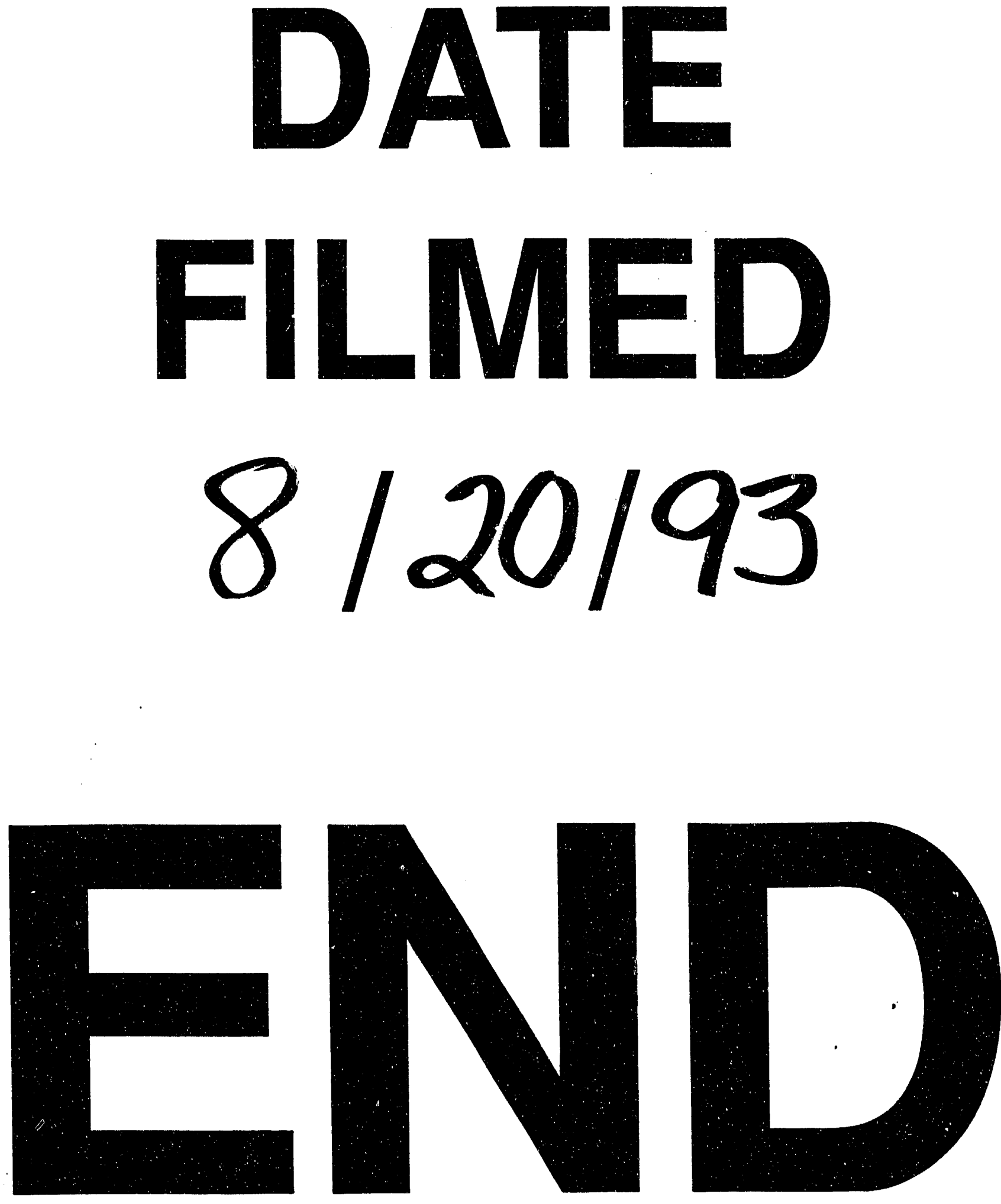


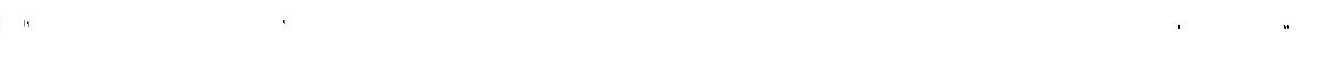

